\title{
Ethical dilemma
}

\section{Retrieving semen from a dead patient}

Although guidelines for retrieving semen from dead patients exist, they may not cover every case. Here, a team of doctors describes the case of a man who underwent orchidectomy after being certified brain dead, and an obstetrician, neurosurgeon, and sociologist debate the ethical issues.

\section{Utilitarianism in the absence of definitive guidelines}

\author{
Michael Swinn, Mark Emberton, David Ralph, Martin Smith, Paul Serhal
}

\section{Case report}

Late at night, after a sudden collapse and grand mal seizure, a 41 year old man was transferred to the intensive care unit of our hospital. He was known to have a right cerebral arteriovenous malformation and had survived two intracerebral haemorrhages (in 1994 and 1996). The patient had had a respiratory arrest before admission, and had been intubated and ventilated before he arrived. His Glasgow coma score was 3/15, and his pupils were fixed and dilated. Computed tomography showed massive intracerebral bleeding. In due course, brain stem death was confirmed according to standard criteria.

The following day the patient's wife came to see us with a paper on which the following was typed: "I confirm that in the circumstances of my death, I give consent to my sperm being saved for my wife to have our children in the future." It was signed by the patient and dated some time the previous year. Since there was no general urology service at the hospital, the uroneurology registrar was contacted for his advice. When he arrived he found that the patient's wife had given consent to multiorgan donation (except corneas, bone, and skin), and arrangements were in place for this to go ahead. Two teams of transplant surgeons were already on their way. The registrar was then given the paper and told that the couple had been attending a fertility clinic where the wife had been assured that her husband had produced normal spermatozoa. None, however, had been stored.

Not having been involved with semen retrieval before, the registrar telephoned a senior urological colleague at another hospital within the trust and after some discussion a consultant urologist with an interest in andrology was contacted and came to the intensive care unit. After considerable debate, which involved consultation with the Human Fertilisation and Embryology Authority, it was decided to proceed with semen retrieval. To establish the presence of viable spermatozoa, the local assisted conception unit was contacted.

The simplest approach, seminal vesicle massage, failed to produce any spermatozoa, and electroejaculation, a technique developed in animal husbandry and the method of choice in spinal injury patients, was not feasible in the time available. Although it had never been described before in these circumstances, we opted to perform an orchidectomy. This was a quick and easy procedure and seemed the most certain way of producing a suitable sample of semen. Light microscopy performed at the bedside showed numerous motile spermatozoa in the fluid obtained from the cut end of the vas. Five vials were prepared for cryopreservation, and the testicular tissue was also processed and frozen for potential subsequent use.

\section{Clinical and ethical problem}

This situation created for us a clinical and ethical dilemma on two main counts. Firstly, we had anxieties about the validity of the consent form as it lacked formality and had not been countersigned by a witness. Secondly, even if the consent were valid, would we be authorised to retrieve semen? On one hand, we were concerned about the possibility of committing a technical assault on the patient, but on the other we were keen to carry out the wishes of the patient and his wife.

An added frustration was the fact that although the couple had been attending a fertility clinic and knew of the husband's underlying vascular abnormality, they had not foreseen that this scenario might occur. It would have been a straightforward matter at that time for a sample of semen to have been stored and for informed consent to be given for its subsequent use. We were also worried about exposing ourselves to a later challenge if we did not carry out the wishes of the patient. The patient's wife was very keen for us to proceed and because we were also aware that by not carrying out retrieval we would effectively be committing an irreversible act, we concluded that the interests of those involved would best be served by the actions we took.

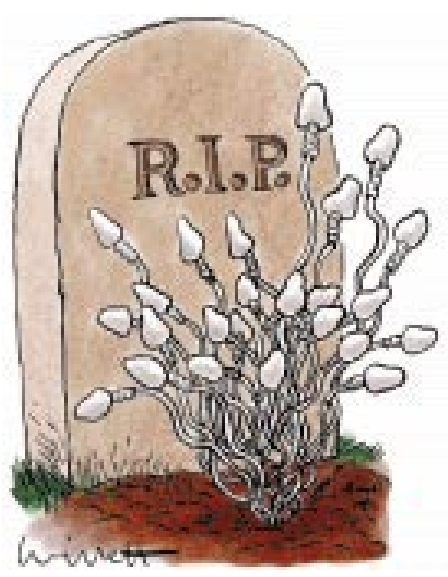

\author{
National Hospital \\ for Neurology and \\ Neurosurgery, \\ London \\ WC1N 3BG \\ Michael Swinn, \\ registrar, department \\ of uro-neurology \\ Martin Smith, \\ consultant, \\ department of \\ surgical intensive care \\ Institute of Urology \\ and Nephrology, \\ London W1P 7PN \\ Mark Emberton, \\ senior lecturer \\ David Ralph, \\ consultant \\ Assisted \\ Conception Unit, \\ University College \\ Hospital, London \\ WC1E 6DB \\ Paul Serhal, \\ head
}

Correspondence to: Mr Swinn

BMJ 1998;317:1583-5 


\section{The patient was assaulted}

Susan Bewley

Department of Obstetrics and Gynaecology, Guy's and St Thomas's Hospital Trust, London SE1 7AE Susan Bewley, obstetrician bewley@globalnet. co.uk doctors' actions?
In this case both the husband's and the wife's wishes were fulfilled-his organs helped many patients and a new life may be created after the fatal tragedy. There is seemingly no victim, so what could be wrong with the

The note was treated as an advance directive, though it did not cover the situation in which the patient found himself. He had considered the creation of a fatherless child using his sperm (which the Human Fertilisation and Embryology Authority regulates), but the collection necessarily included a "touching" by a doctor and is thus an assault without proper consent. Massage, electrical stimulation, and testicular biopsy are all intrusive procedures, albeit minimally so, but we cannot assume that the patient would have agreed to having his testicle removed surgically. Without proper directions the doctors could not know his wishes-nor could his soon to be widow-and they should have refrained, as they had no authority for surgery.

The removal of the other organs is not a relevant comparison. Not only are they covered by specific transplant law but they are for the benefit of other patients unknown to the doctors and thus unable to exert undue influence. However, the wife cannot give proxy consent, particularly as the operation was for her benefit. She is a healthy person-albeit in a tragic situation-with a desire to become pregnant, but owed no obligation of medical assistance by her husband's doctors.

To admit to the weakness of fearing a later (presumably legal) challenge is not a good enough reason to commit the most basic of ethical harms-the invasion of bodily integrity without consent and the treating of one person as a means to another's ends. What legal remedy could the widow have invoked? She could not demand that doctors perform an illegal operation. If the doctors were unsure, they could have suggested that the wife apply for a statutory declaration that the operation would not have been illegal. In the case of caesarean section, courts have given urgent decisions about consent, so time is not an impossible obstacle.

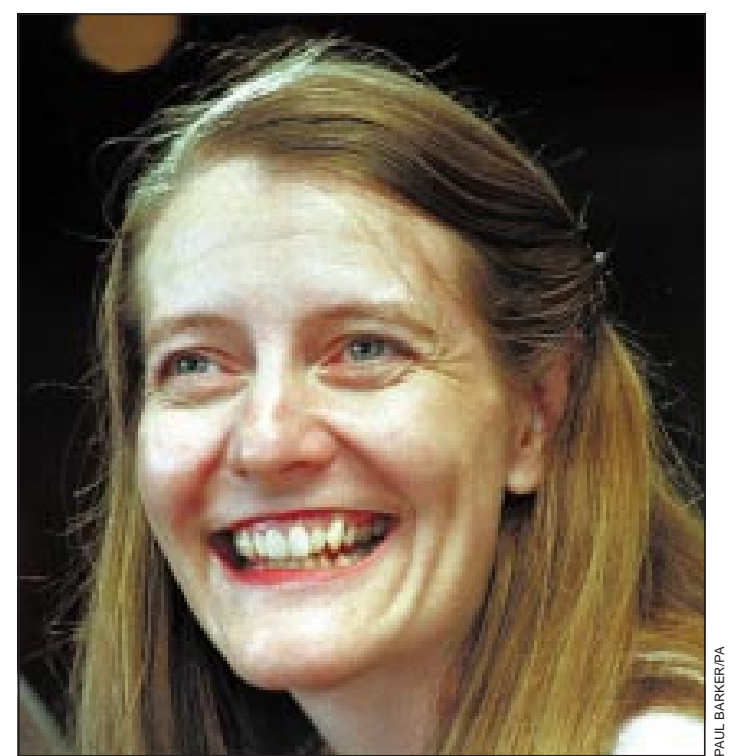

In the public furore about denying Diane Blood access to her dead husband's semen sample, the initial wrong of taking it was overlooked

This is a consequence (and actually a misreading) of the Blood case, where there was no question about consent, though the assault was less intrusive. It is a shame that in the public furore about denying Mrs Blood access to the semen sample once it existed, the initial wrong of taking it was overlooked. Both cases involved pressurised doctors in a rush, which is how medical (and ethical) mistakes are made. Doctors have thus unwittingly sanctioned the use of dead men's bodies for women's gratification. It must be hard in an urgent, emotionally charged situation to deny a wife's wishes, but unless there is the clearest guidance there should be a complete moratorium on such semen retrieval. Doctors must find the courage to say no to assaulting vulnerable, brain dead patients.

\section{Some ethical concerns were ignored}

Boleslav Lichterman

A case of sperm donation from a brain dead patient is not unique-similar cases have been reported recently. ${ }^{1-3}$ The authors argue that this situation created an ethical dilemma for them on two main counts-validity of the consent form as it lacked formality and authorisation of semen retrieval. I share their concerns about the possibility of committing a technical assault on the patient on one hand and the desire to follow the wishes of the patient and his wife on the other. It is frustrating indeed that semen had not been stored during the previous visit to a fertility clinic.

\section{Validity, definition, and benefit}

Although I support the authors' conclusion that the interests of those involved were best served by their actions, I believe that two additional ethical problems are illustrated by this case. Firstly, I question the validity of a consent form signed by someone with a right cerebral arteriovenous malformation complicated by two intracerebral haemorrhages. Patients with lesions of the right hemisphere are often uncritical, and their power to make decisions is impaired. ${ }^{4}$ However, the results of psychiatric assessment are not available here, 
and from a legal standpoint the patient's incapacity can be decided only by a court (at least in Russia). The diagnosis of brain death is a further ethical difficulty. The authors state briefly that "in due course brain stem death was confirmed according to standard criteria." However, standard criteria for brain death do not exist-these vary in different countries. ${ }^{5}$ Although whole brain death remains the standard for determining death in much of the Western world, there are serious discrepancies between the definition of brain death and the criteria used to establish it. ${ }^{6}$

This case also provides an excellent illustration of multiorgan donation preceded by the diagnosis of brain death. ${ }^{7}$ But while organs retrieved from the patient by transplant surgeons were to be used for the benefit of other patients, the retrieved semen was intended for the patient's wife.

\section{Costs and priorities}

Sperm donation and storage at assisted conception units is a costly procedure not covered by the NHS.
This may be the reason why spermatozoa were not stored when the couple attended the fertility clinic. In this case, semen retrieval and cryopreservation were provided free at an NHS hospital. Other couples, faced with the possibility of such a scenario, might elect to follow this precedent for economic reasons. Of course, consultant urological surgeons are free to perform orchidectomies for semen retrieval in brain dead patients provided they have no other work to do and are not under pressure to treat other NHS patients on a waiting list.

1 Iserson KV. Sperm donation from a comatose, dying man. Cambridge $Q$ Healthcare Ethics 1998;7:209-13.

2 Pozda R, Miedema F, Matthews M. Sperm collection in the brain dead patient. Dimens Crit Care Nurs 1996;15:98-104.

3 Webb SM. Raising sperm from the dead. J Androl 1996;17:325-6.

4 Babenkova SV. Clinical syndromes of the right hemispherical lesions in acute stroke. Moscow: Meditsyna, 1971:36.

5 Walker AE. Cerebral death. Munich: Urban and Schwarzenberg, 1985.

6 Bernat JL. A defence of the whole-brain concept of death. Hastings Centre Rep 1998;28:14-23.

7 Surmon OS. Diagnosis of brain death and organ donation. JAMA 1992;268:1859-60.

\title{
An ethic of ambivalence
}

\author{
Deborah Lynn Steinberg
}

The retrieval of organs and tissue from brain dead patients raises a number of ethical issues about which there has been, at best, uncomfortable resolution. These issues include:

- The question of the prior will and intentions of the patient in the event of brain death (and the manner in which these intentions have been signalled)

- The unpredictability of circumstances surrounding retrieval procedures as well as the condition of the patient's body

- The latitude of interpretation that may be exercised legitimately by next of kin, hospital administrators, and doctors.

In the ideal case, the patient's intentions with regard to organ and tissue donation will have been clearly indicated in writing and witnessed, next of kin will fully support the donation, and procedures for retrieval will be routine and without complications. Yet even under such circumstances, organ donation can disturb because of the perceived dehumanisation implicit in the utilitarian treatment of bodies.

In this case, the "ideal" conditions were only partly fulfilled. The patient's consent to his sperm being saved for future use by his wife was indicated in writing, but not witnessed (sperm retrieval in this case was approved by the Human Fertilisation and Embryology Authority). The non-surgical procedure for retrieval of sperm failed, and it became necessary to perform an orchidectomy-a surgical procedure for which no prior consent by the patient had been indicated (or could have been anticipated). Lastly, while multiorgan donation had been an ethically normal practice at this hospital, on consent of the next of kin, the practice had never been extended to semen retrieval.

Swinn et al raised concern both about the informality of the patient's consent and about the possibility that the unauthorised surgical form of sperm retrieval, although seeming to honour the wishes in principle of the patient and his wife, constituted a technical assault. The question of technical assault seems to be obviated by the fact that multiorgan donation was to be performed anyway and involved surgical interventions that the brain dead patient would not benefit from. With regard, however, to the question of the disposition of one's own body post mortem or in the event of brain death, it would seem axiomatic that there must be written prior consent, legally validated by a proper witness. It would be my view that the consent only of next of kin for organ and tissue donation is insufficient. It is not clear in this case whether the patient had agreed in advance to the multiorgan donation for which his wife gave her consent.

If the patient's wife was deemed to have the right to consent to the donation of other organs and tissue, however, there would be no logic in excluding testicular tissue. (The approval granted by the Human Fertilisation and Embryology Authority to perform the sperm retrieval suggests that it was satisfied that the "informal" statement presented to the hospital by the patient's wife did, in fact, represent legitimately the wishes of the patient.)

This case raises a more general point. As with euthanasia, the issue of organ retrieval from brain dead patients raises questions about the possibility of definitive ethical guidelines covering practices that skirt the boundaries of acceptable treatment of human beings. It would seem evident that such cases, more often than not, involve complications that reopen the question of the practice's legitimacy per se. Sometimes, however, ambivalence and discomfort are the appropriate ethical responses. Some discussions, such as this one, need to remain open.

\author{
Department of \\ Sociology, \\ University of \\ Warwick, Coventry \\ CV 7AL \\ Deborah Lynn \\ Steinberg, \\ sociologist \\ sysag@csv.warwick. \\ ac.uk
}

